\title{
A Relevância e o Rigor Científico e Metodológico da Pesquisa Qualitativa em Oncologia
}

doi: https://doi.org/10.32635/2176-9745.RBC.2021v67n2.2187

\author{
The Relevance and Scientific and Methodological Rigor of Qualitative Research in Oncology \\ La Relevancia y el Rigor Científico y Metodológico de la Investigación Cualitativa en Oncología
}

\section{Mario Jorge Sobreira da Silva'; Fernando Lopes Tavares de Lima²}

A pesquisa qualitativa vem ganhando notoriedade há aproximadamente um século, tendo se fundamentado, especialmente, no campo das ciências sociais e humanas ${ }^{1}$. No âmbito da oncologia, desde o início dos anos 1990, tem sido observado um aumento no número de artigos científicos publicados com o uso de metodologias qualitativas ${ }^{2}$. Entretanto, o debate sobre a importância, qualidade, utilidade e validade dos achados provenientes desse tipo de estudo ainda é muito presente. Muitas das críticas se fundamentam pelo desconhecimento de diversos estudantes, profissionais ou pesquisadores, sobre a finalidade dessas pesquisas e quanto ao rigor científico e metodológico empreendidos no seu desenvolvimento.

Considerando os avanços no tratamento do câncer, que visam a ampliar o valor e a qualidade dos regimes terapêuticos, tem se tornado cada vez mais importante compreender as preferências e experiências de pacientes, profissionais e gestores no cuidado em oncologia. No entanto, métodos quantitativos são insuficientes e inadequados para responder a essas e outras perguntas de pesquisas que investigam a compreensão abrangente de fenômenos complexos ${ }^{3}$. É nesse escopo de investigação, por meio do uso de diferentes abordagens teóricas e metodológicas, que se enquadram as pesquisas qualitativas.

Em oncologia, as principais abordagens em estudos qualitativos envolvem, embora não se limitem, as pesquisas etnográficas, fenomenológicas, narrativas, teoria fundamentada em dados e estudos de casos ${ }^{4}$. Em geral, para responder às perguntas de pesquisa, são utilizadas técnicas de observação, entrevistas, grupos focais, análises documentais e de imagens ${ }^{5}$. Todas essas abordagens e técnicas de coletas de dados são particularmente adequadas para atender aos propósitos desse tipo de pesquisa e para responder a questóes de pesquisas voltadas à compreensão dos aspectos envolvidos no cuidado e nos resultados em oncologia.

Os métodos qualitativos podem fornecer uma descrição, exploração ou explicação de um fenômeno de maneira independente ou, ainda, em combinação com dados oriundos de estudos quantitativos ${ }^{4}$. $\mathrm{O}$ foco principal da pesquisa qualitativa está na elucidação de como e por que um fenômeno ocorre, com base nos conhecimentos, perspectivas e prioridades daqueles que estáo sendo estudados e dos acontecimentos no mundo real. Um aspecto central dessa abordagem de pesquisa é a aceitação da incerteza e a autenticidade estabelecida no contexto das interaçóes sociais ${ }^{6}$. Trata-se, portanto, de um trabalho árduo por parte dos pesquisadores, uma vez que as prioridades da pesquisa são sempre estabelecidas a partir da perspectiva dos pesquisados.

Em que pese suas características, é de suma importância que a pesquisa qualitativa esteja devidamente ancorada em uma estrutura teórico-metodológica apropriada ${ }^{7}$. O uso de um referencial teórico consistente irá dar suporte às análises e interpretaçôes dos autores, evitando a adoção do "senso comum". Essa definição a priori deve estar explícita e justificada para que os leitores entendam como os autores se adaptaram e aplicaram as questôes de pesquisa, as escolhas metodológicas e as análises realizadas ${ }^{6}$. No entanto, se o objetivo da pesquisa for desenvolver indutivamente um quadro teórico com base nos dados emergentes, os autores deverão justificar detalhadamente as motivaçóes para não empregar um referencial teórico a priorí. Em suma, é fundamental que as escolhas metodológicas da pesquisa qualitativa estejam alinhadas aos objetivos da pesquisa.

Destaca-se, ainda, que a análise qualitativa de dados não é um simples processo padronizado, mas customizado, que combina as etapas de coleta, organizaçấo, análise e síntese dos dados. No contexto da oncologia, essas análises devem ser transparentes. As técnicas utilizadas devem ser bem descritas e fundamentadas no referencial teórico escolhido. A clareza dessas informaçôes irá permitir aos leitores compreender como conceitos, categorias, dimensôes ou temas

'Instituto Nacional de Câncer José Alencar Gomes da Silva (INCA). Coordenação de Ensino (Coens). Rio de Janeiro (RJ), Brasil. E-mail: mario.silva@inca.gov.br. Orcid iD: https://orcid.org/0000-0002-0477-8595

${ }^{2}$ INCA/Coens. Rio de Janeiro (RJ), Brasil. E-mail: fernando.tavares.lima@gmail.com. Orcid iD: https://orcid.org/0000-0002-8618-7608

Endereço para correspondência: Mario Jorge Sobreira da Silva. Rua Marquês de Pombal, 125, 30 andar - Centro. Rio de Janeiro (RJ), Brasil. CEP $20230-240$. E-mail: mario.silva@inca.gov.br 
emergiram dos dados. As técnicas analíticas primárias mais empregadas nas pesquisas qualitativas em oncologia são: análise de conteúdo, análise narrativa, análise de discurso, análise fenomenológica, análise de estrutura e análise da teoria fundamentada em dados ${ }^{3,4}$. Essas análises podem ser realizadas com o apoio de programas informatizados específicos para essa finalidade, como o $\mathrm{NVivo}^{\circledR}$ e o MAXQDA ${ }^{\circledR}$, ou com o uso de outras ferramentas não específicas, como planilhas e editores de textos ${ }^{4}$.

A chave para se garantir o rigor da pesquisa qualitativa está no desenvolvimento de um plano metodológico capaz de justificar as escolhas realizadas pela equipe de investigação. Na apresentação dos resultados, discussão e conclusão, é necessário que fique claro para os leitores os elementos-chave da pesquisa, como as características dos sujeitos ou documentos investigados, fontes e instrumentos de coleta de dados utilizados, técnicas de coleta e análise $e^{6}$ A intencionalidade e o envolvimento significativo dos pesquisadores, com suas influências potenciais (reflexividade), devem ser devidamente apresentados, garantindo a ética e a transparência necessárias ${ }^{8,9}$. Tais cuidados, na condução e apresentação dos resultados dos estudos qualitativos, contribui para as validades interna e externa da pesquisa, além de aumentar a confiabilidade das análises.

No momento de relatar os resultados da pesquisa qualitativa, dependendo das escolhas metodológicas realizadas, a Revista Brasileira de Cancerologia (RBC) recomenda aos autores o uso do COREQ (Consolidated criteria for reporting qualitative research $)^{10}$ ou do SRQR (Standards for reporting qualitative research) ${ }^{11}$. Essas ferramentas definem padróes para o relato das pesquisas qualitativas, ao mesmo tempo que preservam a flexibilidade necessária aos diferentes paradigmas, abordagens e métodos qualitativos.

Apesar dos desafios metodológicos existentes em realizar estudos qualitativos de qualidade, cabe destacar que os resultados provenientes desse tipo de abordagem metodológica têm sido fundamentais para os avanços no cuidado em oncologia ${ }^{4}$. Para ampliar a aplicabilidade desse tipo de pesquisa, espera-se que os pesquisadores sejam capazes de abordar questóes relevantes e que utilizem todos os recursos disponíveis para garantir o rigor metodológico necessário ao desenvolvimento do conhecimento científico no campo da prevenção e do controle do câncer.

\section{REFERÊNCIAS}

1. Gomes R, Deslandes SF, Moreira MCN. As abordagens qualitativas na Revista Ciência \& Saúde Coletiva (1996-2020). Cienc Saúde Colet. 2020;25(12):4703-14. doi: https://doi.org/10.1590/1413-812320202512.18142020

2. Borreani $\mathrm{C}$, Miccinesi $\mathrm{G}$, Brunelli $\mathrm{C}$, et al. An increasing number of qualitative research papers in oncology and palliative care: does it mean a thorough development of the methodology of research? Health Qual Life Outcomes. 2004;2:7. doi: https://doi.org/10.1186/1477-7525-2-7

3. Strang P. Qualitative research methods in palliative medicine and palliative oncology-an introduction. Acta Oncol. 2000;39(8):911-7. doi: https://doi.org/10.1080/02841860050215873

4. Hannum SM, Dy SM, Smith KC, et al. Proposed criteria for systematic evaluation of qualitative oncology research. J Oncol Pract. 2019;15(10):523-9. doi: https://doi.org/10.1200/JOP.19.00125

5. Thorne S. Data analysis in qualitative research. Evid Based Nurs. 2000;3(3):68-70. doi: http://doi.org/10.1136/ebn.3.3.68

6. Minayo MCS. Análise qualitativa: teoria, passos e fidedignidade. Cienc Saúde Colet. 2012;17(3):621-6. doi: https:// doi.org/10.1590/S1413-81232012000300007

7. Minayo MCS, Costa AP. Fundamentos teóricos das técnicas de investigaçáo qualitativa. Rev Lusof Educ. 2018;40(40):139-53.

8. Peter E. The ethics in qualitative health research: special considerations. Cienc Saúde Colet. 2015;20(9):2625-30. doi: https://doi.org/10.1590/1413-81232015209.06762015

9. Minayo MCS, Guerriero ICZ. Reflexividade como éthos da pesquisa qualitativa. Cienc Saúde Colet. 2014;19(4):110312. doi: https://doi.org/10.1590/1413-81232014194.18912013

10. Tong A, Sainsbury P, Craig J. Consolidated criteria for reporting qualitative research (COREQ): a 32-item checklist for interviews and focus groups. Int J Qual Health Care. 2007;19(6):349-57. doi: https://doi.org/10.1093/intqhc/mzm042

11. O'Brien BC, Harris IB, Beckman TJ, et al. Standards for reporting qualitative research: a synthesis of recommendations. Acad Med 2014;89(9):1245-51. doi: https://doi.org/ 10.1097/ACM.0000000000000388 